\title{
ANALYSIS OF RISK FACTORS ASSOCIATED WITH STROKE IN YOUNG ADULTS : A PROSPECTIVE STUDY
}

\author{
HOSSAIN MZ ${ }^{1}$, AHMED SU ${ }^{2}$, SARDER MH ${ }^{1}$, DASGUPTA R ${ }^{1}$, DAS A ${ }^{1}$, \\ SARKER RN ${ }^{6}$, AZAD KAK $^{3}$, ARIF SM $^{5}$, MOHAMMAD QD ${ }^{4}$
}

\begin{abstract}
:
Objective: Stroke in young patients is not common. The purpose of this study was to find out the risk factors of stroke in young adults in Bangladesh and to compare with different other studies.

Methods: 85 young adult cases of stroke patients with the age ranging from 14 to 45 years, admitted in different medical units of Dhaka Medical College Hospital from January 2008 to July 2009, were studied. The risk factors for stroke and the distribution of stroke types were analyzed.

Results: The specific age group (14-45 years old) included 6\% of patients of all ages admitted for stroke. Among 85 patients, cerebral infarction was diagnosed in 52 patients (61\%). Intracerebral hemorrhage (without trauma) was diagnosed in 25 patients (29.4\%). Subarachnoid hemorrhage was found in 7 young patients (8.24\%). The most common risk factors for ischemic stroke were hypertension (57.68\%), hypercholesterolemia (38.46\%), diabetes (34.61\%), smoking (32.69\%), premature atherosclerosis (11.54\%). Regarding aetiology of cerebral infarction, the majority due to cardiogenic emboli (75\%). The use of oral contraceptives, pregnancy, SLE, migraine and moya moya disease were infrequent causes of cerebral infarction. In ischemic stroke, the ratio of cardiogenic emboli \& other factors were 3:1. The most common risk factors for hemorrhagic stroke were hypertension (63.63\%), hypercholesterolemia (39.39\%), diabetes (36.54\%) \& smoking (33.33\%).

Conclusion: Hypertension, Diabetes, tobacco smoking, premature atherosclerosis \& rheumatic valvular heart disease are growing problems in the developing countries. According to our finding, identification \& treatment of hypertension, dyslipidaemia, diabetes \& rheumatic valvular heart disease \& cessation of smoking are proper measures to prevent morbidity \& mortality.
\end{abstract}

Key words: Risk factors, stroke, young adult.

J Dhaka Med Coll. 2009; 18(2) : 95-100

\section{Introduction:}

Stroke is a major cause of morbidity \& mortality ${ }^{1}$. It is one of the most common neurological disorder $\&$ third common cause of death worldwide ${ }^{2}$. The world health organization defined stroke as a syndrome of rapidly developing clinical sign of focal or global disturbance of cerebral function, persisting for at least 24 hours or leading to death with no apparent cause other than vascular origin ${ }^{3}$.

Stroke may be ischemic or hemorrhagic \& usually occurs in the elderly and middle aged.
Stroke in young adults are relatively uncommon. Stroke in young adults include a wide variety of disorders those are less frequent in older age groups. Though there are some overlapping in the risk factors between these two groups but there are some clearly distinct risk factors for ischemic \& hemorrhagic strokes in young adults; e.g. congenital heart disease (patent foramen ovale), valvular heart disease, family history of stroke, premature atherosclerosis, connective tissue disease with vasculitis, hematological variables, smoking,

1. Assistant Professor of Medicine, DMCH.

2. Lecturer, Comilla Medical College.

3. Professor of Medicine, DMCH.

4. Professor of Neuromedicine, DMCH.

5. Associate Professor of Medicine, DMCH.

6. Assistant Professor of Radiology, DMCH.

Correspondence : Dr. Mohammad Zaid Hossain. 
drug abuse, some genetic disease, Coagulopathy, AVM, aneurysm, \& in female oral contraceptive pill, pregnancy, postpartum state, migraine, etc ${ }^{4,5}$.

This suggests that it should be possible to make a considerable impact on the stroke incidence in young adults by reducing the prevalence of relevant risk factors in the population by screening or case finding for high risk individuals to whom preventive treatment may be offered.

In term of risk factors stroke in young adults is remarkably heterogenous \& often a diagnostic challenge. Western reports ${ }^{6,7}$ show that in this age group, cardio embolisation $\&$ non atherosclerotic vasculopathies are relatively important risk factors of ischemic stroke as compared to atherosclerotic vasculopathy \& small artery occlusion. It is important to find out the etiologic factors \& management of these factors adequately for appropriate prevention \& recurrence. Type of stroke in young adults may influence the outcome \& may have a dramatic impact in the quality of life in survivors ${ }^{8}$.

Clinical features \& investigations for diagnosis of stroke are similar in the both group. For identification of causes $\&$ risk factors there are additional investigations depending on the history \& clinical examination.

Stroke is a leading cause of death in developing countries like Bangladesh, afflicting individuals at a young age ${ }^{9}$. We have studied the risk factors $\&$ stroke subtype of stroke in young adults in Bangladesh \& compared our results with previous reports.

\section{Methods:}

This study is based on the data from Dhaka Medical College Hospital, Dhaka, Bangladesh. We reviewed the medical records of 85 young patients aged from 14 to 45 years who were admitted to the hospital and were diagnosed as stroke. The study period was January 2008 to July 2009.

The following stroke risk factors were assessed: sex, hypertension (defined by preadmission history and medical records), diabetes mellitus, current cigarette smoking, alcohol consumption, drug abuse, hypercholesterolemia, coronary artery disease, transient ischemic attack (TIA, neurological deficit lasting $<24$ hours), migraine (diagnosed in accordance with the criteria of the International Headache Society), use of oral contraceptives, pregnancy, puerperium, eclampsia, coagulation abnormality, AVM, and aneurysm.

The severity of the neurological deficit on admission was assessed using the National Institutes of Health stroke scale (NIHSS).

All patients were investigated using a standard protocol including complete blood count and ESR, serum creatinine, serum electrolytes, random blood sugar, 12 lead electrocardiography, cranial computed tomography (CT) and/or conventional magnetic resonance imaging (MRI), lipid profile, echocardiography, ANA, anti ds DNA, c-ANCA, p-ANCA, antiphospholipid antibodies, C reactive protein, VDRL, cerebral angiography, doppler study of neck vessels.

All patients were monitored closely throughout their hospital admitted period.

Our study excluded patients with venous infarction \& those with ischemic strokes caused by complications arising from trauma $\&$ cardiac surgery.

Statistical analysis:

Statistical analysis was carried out using SPSS 12.0 version.

\section{Results:}

We studied 85 patients of stroke aged 14 to 45 years in one and half years. This specific age group comprised 6.02 percent of the 1416 patients of all ages stroke patients admitted in the hospital during the study period. The Age and sex distribution of the patients is shown in the table below.

Table 1 shows the age and sex distribution of the patients. 27.06 percent were 14 to 25 years of age, $29.41 \%$ were in 26 to 35 years of age, and $43.53 \%$ were 36 to 45 years of age. 
Thus, there is a rising trend of the incidence of cerebrovascular disorders in general as the patients get older, even in this young adult group.

The mean age of the patients was 31.88 years. Male female ratio was 1.13:1

Table-I

Age and sex distribution of the Patients

\begin{tabular}{lccc}
\hline Total number: & \multicolumn{3}{c}{$\mathrm{n}=85$} \\
\hline Age & $14-25$ & 23 & 27.06 \\
& $26-35$ & 25 & 29.41 \\
& $36-45$ & 37 & 43.53 \\
Sex & male & 45 & 52.94 \\
& female & 40 & 47.06 \\
\hline
\end{tabular}

Table II shows the data down according to diagnosis. The most common diagnosis was cerebral infarction, followed by intracerebral hemorrhage, subarachnoid hemorrhage, and aneurysm.
Table-II

Types of stroke

\begin{tabular}{lcc}
\hline Type of stroke & No. & percent \\
\hline Subarachnoid hemorrhage & 7 & 8.24 \\
Aneurysm & 1 & 1.18 \\
intracerebral hemorrhage & 25 & 29.4 \\
Cerebral infarction & 52 & 61.18 \\
\hline
\end{tabular}

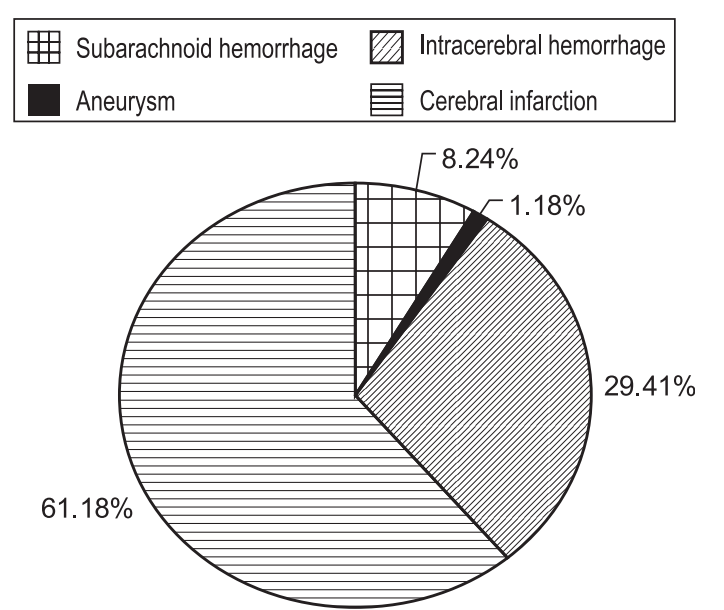

Risk factors for infarctive stroke were distributed as reported in Table-III.

Table-III

Risk factors of stroke (infarction)

\begin{tabular}{lccc}
\hline Risk Factors & \multicolumn{3}{c}{ Infarction(n $=52)$} \\
\cline { 2 - 4 } & $\begin{array}{c}\text { Male } \\
\text { (percent) }\end{array}$ & $\begin{array}{c}\text { Female } \\
\text { (percent) }\end{array}$ & Total \\
\hline Hypercholesterolemia $(6.5 \mathrm{mmol} / \mathrm{L})$ & $17(32.69)$ & $3(5.77)$ & $20(38.460$ \\
Hypertension & $21(40.38)$ & $9(17.30)$ & $30(57.680$ \\
Current smoking & $10(19.23)$ & $7(13.46)$ & $17(32.690$ \\
Diabetes mellitus & $10(19.23)$ & $8(15.38)$ & $18(34.61)$ \\
Oral contraceptive use & $0(0)$ & $2(3.85)$ & $2(3.85)$ \\
Migraine & $0(0)$ & $1(1.92)$ & $1(1.92)$ \\
Premature atherosclerosis & $3(5.77)$ & $3(5.77)$ & $6(11.54)$ \\
Toxaemia of pregnancy & $0(0)$ & $0(0)$ & $0(0)$ \\
Pregnancy & $0(0)$ & $2(3.85)$ & $2(3.85)$ \\
SLE & $0(0)$ & $1(1.92)$ & $1(1.92)$ \\
Moyamoya disease & $0(0)$ & $1(1.92)$ & $1(1.92)$ \\
Mitral valve prolapse & $3(5.77)$ & $1(1.92)$ & $4(7.70)$ \\
Mitral stenosis & $15(28.84)$ & $24(46.15)$ & $39(74.99)$ \\
Patent foramena ovale & $3(5.77)$ & $1(1.92)$ & $4(7.70)$ \\
Aneurysm & $0(0)$ & $0(0)$ & $0(0)$ \\
Family history of stroke & $7(13.46)$ & $4(7.70)$ & $110(21.16)$ \\
\hline
\end{tabular}


Risk factors for Hemorrhagic stroke were distributed as reported in Table-IV.

Table-IV

Risk factors of stroke (hemorrhage)

\begin{tabular}{lccc}
\hline Risk Factors & \multicolumn{3}{c}{ Hemorrhage $(\mathrm{n}=33)$} \\
\cline { 2 - 4 } & Male (percent) & Female (percent) & Total(percent) \\
\hline Current smoking & $10(30.30)$ & $1(3.03)$ & $11(33.33)$ \\
Hypertension & $14(42.42)$ & $7(21.21)$ & $21(63.63)$ \\
Diabetes mellitus & $8(24.24)$ & $4(12.30)$ & $12(36.54)$ \\
Hypercholesterolemia $(6.5 \mathrm{mmol} / \mathrm{L})$ & $7(21.21)$ & $6(18.18)$ & $13(39.39)$ \\
Oral contraceptive use & $0(0)$ & $0(0)$ & $0(0)$ \\
Migraine & $0(0)$ & $0(0)$ & $0(0)$ \\
Premature atherosclerosis & $0(0)$ & $1(3.03)$ & $1(3.03)$ \\
Toxaemia of pregnancy & $0(0)$ & $2(6.06)$ & $2(6.06)$ \\
Pregnancy & $0(0)$ & $0(0)$ & $0(0)$ \\
SLE & $0(0)$ & $0(0)$ & $0(0)$ \\
Moyamoya disease & $0(0)$ & $0(0)$ & $0(0)$ \\
Mitral valve prolapse & $0(0)$ & $0(0)$ & $0(0)$ \\
Mitral stenosis & $0(0)$ & $0(0)$ & $0(0)$ \\
Patent foramena ovale & $0(0)$ & $0(0)$ & $0(0)$ \\
Aneurysm & $4(12.30)$ & $1(3.03)$ & $5(15.33)$ \\
\hline
\end{tabular}

Table 5 shows the aetiological factors of cerebral infarction in 52 young patients. Most patients $(75 \%)$ had cardiac disease followed by premature atherosclerosis. SLE, Oral contraceptives, Pregnancy, Migraine, Moyamoya disease were infrequent sole causes of cerebral infarction in the absence of other risk factors and these comprised $25 \%$ of ischaemic stroke. In ischaemic stroke, the ratio of cardiogenic emboli and other factors was 3:1

Table-V

Aetiological factors of cerebral infarction in 52 young patients

\begin{tabular}{lcc}
\hline Factors & \multicolumn{2}{c}{ Patients } \\
\cline { 2 - 3 } & No. & Percent \\
\hline Cardiac & 39 & 75 \\
$\quad$ Mitral stenosis & 26 & \\
Infective endocarditis & 4 & \\
Mitral valve Prolapse & 4 & \\
Congenital heart disease & 4 & \\
Aortic valve defect & 1 & \\
Atherosclerotic occlusive disease & 6 & 11.54 \\
Haematologic & 5 & 9.62 \\
SLE & 1 & \\
Oral contraceptives & 2 & \\
$\quad$ Pregnancy & 2 & \\
Migraine & 1 & 1.92 \\
Moyamoya disease & 1 & 1.92 \\
\hline
\end{tabular}

\section{Discussion:}

In our retrospective study, young patients comprised $6 \%$ of all patients admitted with stroke, both hemorrhagic and non hemorrhagic, 1.5 times that in the National Survey of Stroke.

In the overall patient group presented here, there is a rising trend in the incidence of cerebrovascular disease with age and this agrees with the statement of Kurtzke. ${ }^{10}$

In this study, the most common diagnosis was cerebral infarction (61.18\%), followed by intracerebral hemorrhage (29.4\%), subarachnoid hemorrhage $(8.24 \%)$, and aneurysm (1.18\%).

Among 52 patient of cerebral infarction, we have found that hypertension (57.68\%) is the leading risk factor, followed by hypercholesterolemia (38.46\%), diabetes mellitus (34.61\%), current smoking $(32.69 \%)$, family history of stroke $(21.16 \%)$, premature atherosclerosis $(11.54 \%)$.

Kristensen found, Spontaneous arterial dissection as the leading probable etiology of stroke in young patients in Western countries $^{11}$. In Bangladesh, in our study, no arterial dissection case was found. 
Patent foramen ovale $\&$ atrial septal aneurysm are causes of stroke $(28 \%)$ in Sweden ${ }^{11}$, whereas in Bangladesh we have found 4 cases $(7.69 \%)$ of Patent foramen ovale as a cause of cerebral infarction.

A growing body of data suggests that there are important differences in the distribution of occlusive vascular disease between races. Atherosclerosis of the larger extracranial arteries is more prevalent in whites, whereas occlusive disease of the intracranial arteries is more often seen in patients of black or oriental origin $12,13,14$.

The most common cause of significant extracranial stenosis is dissection, and the most common cause of significant intracranial stenosis is presumed to be premature atherosclerosis ${ }^{15}$. In our study, in Bangladesh we have found $11.54 \%$ patients of premature atherosclerosis as a risk factor of ischemic stroke in young patients.

A cardiogenic cerebral embolus is one of the most common causes of stroke in the young, accounting for up to one third of the cases ${ }^{16,17}$.

In our series, $75 \%$ of the cases of cerebral infarction were of cardiac origin.

The use of oral contraceptives is associated with a 9 fold increased risk of cerebral infarction in women ${ }^{18}$. The Collaborative Group for the Study of Stroke in Young Women found that the risk of stroke with the use of oral contraceptives rose sharply in women with hypertension or migraine and those who were heavy smokers ${ }^{19,20}$. Oral contraceptives alter platelet aggregation, enhance antithrombin III activity, decrease serum antithrombin levels, and increase the levels of certain coagulation factors, especially factor VII. ${ }^{7}$ In our series, $3.85 \%$ of the women with cerebral infarction were using oral contraceptives when their strokes occurred, but only two had strokes attributable solely to the oral contraceptives. It must be remembered that pregnancy increases the risk of ischemic events by approximately 13 times $^{20}$.

Cerebral infarction is a potential complication of migraine headaches. The incidence of migraine among young patients with stroke varies little from that among the general population (approximately 1S-30\%) 21,22,23. Spaccavento and Solomon ${ }^{24}$ found a $27 \%$ incidence of migraine among young adults with stroke and attributed all of the strokes to migraine.

In our series, 1 patients $(1.92 \%$ of those with cerebral infarction) had a history of migraine. Mechanisms by which migraine may produce stroke include vasospasm and/or arteriopathy, embolism, and platelet abnormalities ${ }^{21,22,23}$.

\section{Conclusion:}

We had some limitations such as, we did not do magnetic resonance angiography. Another limitation is use of single occasion measurements of risk factors. Besides limitations, our study raises the possibility that accelerated hypertension, diabetes, premature atherosclerosis (occlusive disease of the intracranial arteries) may be the underlying substrate rather than risk factors like arterial dissection which is more common in western countries ${ }^{11}$. Valvular heart disease is common in western countries ${ }^{25} \&$ also in developing countries like Bangladesh. Tobacco smoking ${ }^{26}$, diabetes, hypertension ${ }^{27} \&$ premature atherosclerosis are growing problems in the developing countries. According to our finding, identification \& treatment of hypertension, rheumatic valvular heart disease, dyslipidemia, diabetes mellitus \& cessation of smoking are proper measures for reducing stroke in young adults in developing countries of south Asia.

\section{Refernces:}

1. Bergen DC. The world wide burden of neurologic disease. Neurology 1996; 47:21-5

2. ADAMS RD, Victor M. Principles of neurology, $15^{\text {th }}$ edition, USA McGraw Hill. 1993: p. $716-717$

3. Aho K, Harmsen P, Hatano S, Marguarsden, strasser T. Cerebrovascular disease in the community: results of WHO collaborative study. Bull, World Health Org, 1980; 58; 113-30

4. Wiebers DO, Feigin VL, Brown RD. 'Cerebrovascular Disease in children \& young adult' in: Handbook of stroke. Philadelphia, PA: Lippincott-Raven; 1977, p. 237-43 
5. J Rohr, S Kittner, B Feeser. Traditional risk factors $\&$ ischemic stroke in young adults: The BaltimoreWashington cooperative young stroke study(July 96), archives of N, JAMA vol 53, no 7

6. Kristensen B, Malm J, Carlberg B et al. 'Epidemiology \& etiology of ischemic stroke in young adults aged 18-44 years in Northern Sweden' (1997). Stroke;28:1702-9

7. BOGOUSSLAVSKY J, peierre P. 'Ischemic strokes under age 45.' Neurolclin 1992, 10: 113-24

8. Walker AE, Robins M, Weinfeld FD: clinical findings of stroke 1981;12:1-15

9. K Lipska, $\mathrm{P} N$ Sylaja, $\mathrm{P}$ K Sarma, K R Thankappan(2007). 'Risk factors for acute ischemic stroke in young adults in south India'. JNNP 78: 959-63

10. Kurtzke JF: Epidemiology of Cerebrovascular Disease. Springer, 1969

11. Kristensen B, Malm J, Carlberg B et al. 'Epidemiology \& etiology of ischemic stroke in young adults aged 18-44 years in Northern Sweden' (1997). Stroke;28:1702-9

12. Caplan LR, Gorelick PB, Hier DB. 'Race.sex \& occlusive cerebrovascular disease a review' (1986). Stroke ; 17: 648-55

13. Feldmann E, Daneault N, Kwan E. 'Chinese white differences in the distribution of occlusive cerebrovascular disease (1990). Neurology; 40:1541-45

14. Leung SY, Ng Th. 'Pattern of cerebral atherosclerosis in Hong Kong Chinese: severity in intracranial \& extracranial vessels (1993). Stroke; 24: 779-86

15. Tsong-Hai Lee, Wen-Chuin Hsu. 'etiologic study of young ischemic stroke in Taiwan' (2002). Stroke; 33: 1950-55
16. Hart RG, Freeman GL: Stroke in young peopleThe heart of the matter. West J Med 1987;146:596597

17. Hachinslri V, Norris JW: The young stroke, in The AcuteStroke. Contemporary Neurology Series, vol 27. Philadelphia, FA Davis Co, 1985, pp 141163

18. Collaborative Group for the Study of Stroke in Young Women: Oral contraception and increased risk of cerebral ischemia or thrombosis. $N$ Engl $J$ Med 1973;288:871-78

19. Collaborative Group for the Study of Stroke in Young Women: Oral contraceptives and stroke in young women, associated risk factors. JAMA $1975 ; 231: 718-22$

20. Wiebers DO: Ischemic cerebrovascular complications of pregnancy. Arch Neurol 1985;42:1106-13

21. Henrich JB: The association between migraine and cerebral stroke in young adults. Headache 1984;24:19-22

22. Vascular events: An analytic review. / Chronic Dis 1987; 25. Rothrock JF, Walicke P, Swenson MR, Lyden PD, Logan WR: 40:329-335 Migrainous stroke. Arch Neul 1988;45:64-67

23. Henrich JB: Sandercock PAG, Warlow CP, Jones $\mathrm{JN}$ : 'Stroke \& migraine in Oxfordshire community'. 1986;45:64-67

24. Gorelick PB: Alcohol \& Stroke. Curr concepts Cerebrovascular dis (1986) Stroke; 21: 21-25

25. H Bevan, K Sharma, W Bradley. 'Stroke in young adults' (1990). Stroke; 21:382-86

26. Jha P, Chaloupka FJ, the economics of global tobacco control' BMJ. 2000; 321: 358-61

27. Asia Pacific Cohort Studies Collaboration. The effects of diabetes on the risks of major cardiovascular disease $\&$ death in the Asia Pacific region. Diabetes care 2003;26:360-6 